\title{
Utilizing nonlinear ELF generation in modulated ionospheric heating experiments for communications applications
}

\author{
G. Jin, ${ }^{1}$ M. Spasojevic, ${ }^{1}$ M. B. Cohen, ${ }^{1}$ and U. S. Inan ${ }^{1,2}$ \\ Received 29 September 2012; revised 3 December 2012; accepted 9 January 2013; published 28 February 2013.
}

[1] Modulated high-frequency heating of the $D$ region ionosphere near the auroral electrojet can generate extremely low frequency (ELF; $3 \mathrm{~Hz}-3 \mathrm{kHz}$ ) radio waves. The modulated heating process is nonlinear and generates harmonics at integer multiples of the ELF modulation frequency. Quaternary phase shift keying, a digital modulation technique is applied to ELF waves to demonstrate transmission of digital data. Data were successfully decoded at a nearby receiver and the bit error rate computed. Square wave modulation of the high-frequency heater results in stronger signals and hence a smaller bit error rate. Simulations of the communication system using ELF waveforms and noise signals derived from ELF observations are also conducted. These simulations show that using higher harmonics of the ELF signal to improve the signal-to-noise ratio can reduce the bit error rate, although only when these harmonics are below $\sim 4.5 \mathrm{kHz}$ because of radio atmospherics (sferics) generating strong impulsive noise at higher frequencies.

Citation: Jin, G., M. Spasojevic, M. B. Cohen, and U. S. Inan (2013), Utilizing nonlinear ELF generation in modulated ionospheric heating experiments for communications applications, Radio Sci., 48, 61-68, doi:10.1002/rds.20014.

\section{Introduction}

[2] Extremely low frequency (ELF; $0.3-3 \mathrm{kHz}$ ) and very low frequency (VLF; $3-30 \mathrm{kHz}$ ) waves have properties that are useful in certain communications applications. These waves can propagate globally in a waveguide formed between the $D$ layer ionosphere and the ground, allowing for reliable communications without depending on line of sight or satellite relays. Waves in this frequency range can also penetrate into sea water and be used to communicate with submarines. However, ELF/VLF waves are difficult to generate because the long wavelengths require long antennas to transmit efficiently. Operational VLF transmitters in the $20 \mathrm{kHz}$ range use large vertical masts. Although these masts are much shorter than a wavelength, reactive elements can be added to the antenna to tune them so they operate efficiently at the transmission frequency. However, this restricts the frequency range over which the transmitter can operate. To transmit at the ELF frequencies needed to communicate with deeply submerged submarines, very large horizontal antennas must be used. The Wisconsin and Michigan transmitters for the United States Navy ELF program and the Russian Zevs transmitter use grounded antennas between 40 and $60 \mathrm{~km}$ long [Velikhov et al., 1998]. These antennas needed to be located in areas with very low ground conductivity deep into the Earth's crust. Otherwise,

\footnotetext{
${ }^{1}$ Department of Electrical Engineering, Stanford University, Stanford, California, USA.

${ }^{2}$ Koç University, Istanbul, Turkey.

Corresponding author: G. Jin, Department of Electrical Engineering, Stanford University, Stanford, California, USA. (gj36@stanford.edu)

(C)2013. American Geophysical Union. All Rights Reserved. 0048-6604/13/10.1002/rds.20014
}

induced ground currents flow beneath the antenna near the Earth's surface, largely cancelling the antenna's radiated fields.

[3] Modulated ionospheric heating is an alternative technique for generating ELF/VLF waves at frequencies below $\sim 10 \mathrm{kHz}$. A high-power, high-frequency (HF, 3-10 MHz) beam is directed upward, and the transmitted wave energy is absorbed by electrons in the $D$ region ionosphere near $80 \mathrm{~km}$ in altitude, heating the plasma and altering the conductivity. This modified conductivity locally alters the large-scale horizontal ionospheric current systems, which are driven by thermal or magnetospheric convection. Amplitude modulation of the heating at ELF/VLF frequencies results in modulated currents that radiate ELF/VLF waves. Modulated heating experiments include those described by [Getmantsev et al., 1974; Kapustin et al., 1977; Rietveld et al., 1987; Barr and Stubbe, 1984; Milikh et al., 1999]. The High Frequency Active Auroral Research Program (HAARP) located in Gakona, Alaska, is currently the world's most powerful ionospheric heater with $3.6 \mathrm{MW}$ of transmitted power and $>1$ GW ERP. The large amount of power and flexibility of HAARP has led to many scientific studies on the interactions between waves and plasmas in the ionosphere and magnetosphere [e.g., Moore et al., 2006; Gotkowski et al., 2008] as well as novel modulation techniques that result in stronger ELF generation [Cohen et al., 2010a].

[4] Several studies have investigated the challenges of communicating at ELF/VLF frequencies, primarily the impulsive noise environment. The dominant source of noise at ELF/VLF frequencies is from lightning discharges, which generate an impulsive signal known as a radio atmospheric or sferic. Although in the analysis of many (non-ELF/VLF) communication systems noise can be treated simply and 
accurately with a white Gaussian model, this assumption is not valid in the ELF/VLF band. (Ziemer [1967] and Hall [1966]) analyzed the bit error rate (BER) performance of communications receivers optimized for white Gaussian noise (described below) in an impulsive noise environment. The measured performance is substantially worse compared with expectations from Gaussian noise particularly at high signal-to-noise ratios. This is a result of the fact that a single strong impulse can cause an error in decoding even a very strong signal. Several other works have proposed nonlinear receivers which attempt to de-emphasize parts of signals that are "too" strong [Hall, 1966; Bernstein et al., 1974; Ingram, 1984]. Kassam [1988], Ch. 3, and the references therein show examples of the behavior of ELF noise and detecting signals in this noise environment. Bernstein et al. [1974] provides a comprehensive overview of the experimental predecessor to the Navy ELF program, including a discussion of generation and propagation of the ELF waves, the design of the signal structure and receiver, and the successful transmission of a message with a $76 \mathrm{~Hz}$ carrier that was received onboard a submerged submarine in the Atlantic Ocean.

[5] These past studies have thoroughly addressed the challenges of impulsive noise, which affects the reception of ELF waves generated by both large antennas and modulated ionospheric heating. However, modulated ionospheric heating has the unique property of strong nonlinear distortion resulting in generation of frequency components that were not present in the original ELF modulation signal due to the nonlinearity between the heating power and the changes in the plasma conductivity [e.g., Tomko, 1981; James, 1985]. These frequency components, or harmonics, are at integer multiples of the fundamental ELF frequency. [Jin et al., 2012] attempted to use nonsinusoidal waveforms so that the generated ELF fields are sinusoidal after distortion, but these waveforms are sensitive to the highly variable ionospheric electron density profile and are also less efficient than square wave modulation although $>15 \%$ of the ELF energy is outside the fundamental frequency. Thus, it is important to consider how that energy can be used to improve the performance of the system. This can be challenging because the characteristics of the ELF/VLF noise environment change with frequency. Figure 1 shows a spectrogram of ELF transmissions from HAARP. The fundamental is at $1510 \mathrm{~Hz}$, and there are several harmonics visible. The noise environment below $\sim 4.5 \mathrm{kHz}$ appears mostly Gaussian, with occasional impulses from nearby lightning strikes. However, the fourth and higher harmonics are exposed to a much more impulsive environment with many sferics. At higher frequencies, sferics can propagate in waveguide modes with less attenuation [e.g., Budden, 1961, p. 34], and sferics propagating over very long distances in the Earth-ionosphere waveguide contribute to the higher noise levels.

[6] In this article, we examine the results of experiments to transmit digital data using quaternary phase shift keying (QPSK; described below) on an ELF carrier generated via modulated ionospheric heating at HAARP. This is the first time such results have been published. We show the measured BERs as a function of signal-to-noise ratio at several bit rates and ELF frequencies. We then examine how using more of the harmonic content of the signal can improve the BER because of the higher signal strength available from the energy in the harmonics. We then present results from

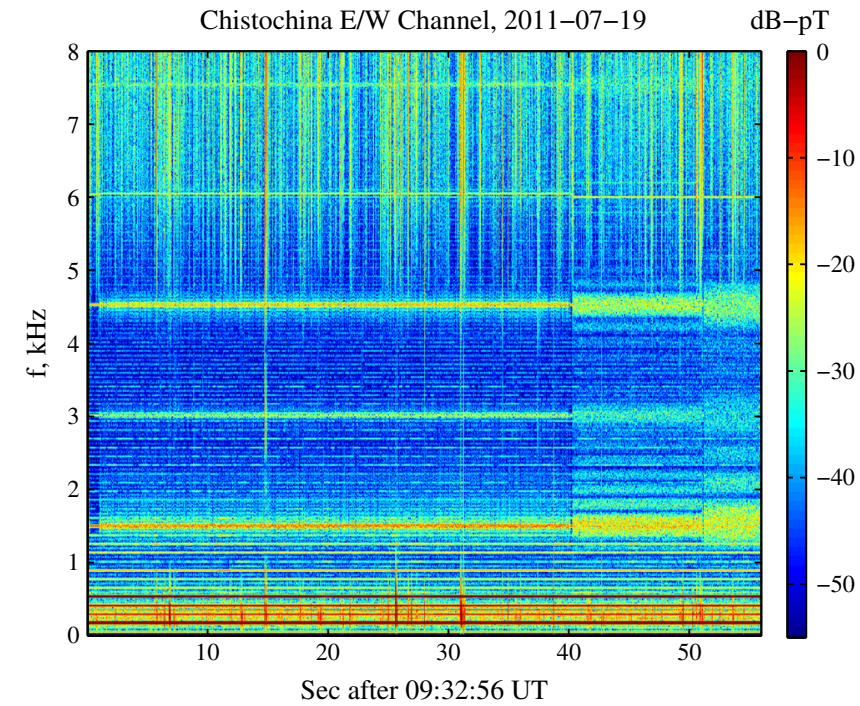

Figure 1. Spectrogram showing transmission of three packets using square wave modulation at $1510 \mathrm{~Hz}$ at bit rates of 100, 400, and 800 bps. Higher bit rates use more bandwidth. Harmonics are clearly visible at integer multiples of $1510 \mathrm{~Hz}$.

computer simulations of the encoding, noise addition, and decoding process, which provide more reliable measurements of the BER because these simulations can be repeated as often as necessary, whereas experimental time at the HAARP transmitter is limited. Finally, we compare the experimental and simulation results with mathematical models from previous works to better characterize the BER of a communications system using an ELF carrier with many harmonics in an impulsive ELF noise environment.

\section{Phase Shift Keying}

[7] The modulation technique used in this article is QPSK or 4-PSK. Digital data are divided into pairs of bits, which are either $00,01,11$, or 10 . To transmit the bits 00 , a symbol, such as a sinusoid of fixed duration, is used. The other bit pairs are represented with a sinusoid phase shifted at 90, 180 , and 270 degrees relative to the symbol representing 00 . The symbol does not necessarily need to be a sinusoid, as modulated heating experiments have shown that other types of heading waveforms such as square waves are more efficient in generating ELF waves [Jin et al., 2012]. Nonsinusoidal waveforms will have harmonic content that increases the bandwidth used by the transmission. However, even sinusoidal modulation generates harmonic content due to the nonlinear conversion between the HF power waveform and the changes in the plasma conductivity. Thus, it may be advantageous to use square waves as the QPSK symbol to take advantage of the greater generated power at the expense of an increase in harmonic content. In this case, the symbols representing 01,11 , and 10 are circularly shifted by a quarter, a half, and three quarters of a fundamental ELF period relative to the symbol representing 00. At the fundamental frequency, the four symbols still have phase shifts of $0^{\circ}, 90^{\circ}, 180^{\circ}$, and $270^{\circ}$. The phase shifts at the higher harmonics are integer multiples of the phase shifts in the fundamental (e.g., $0^{\circ}, 180^{\circ}, 0^{\circ}$, and $180^{\circ}$ in the second 
harmonic). Thus, evenly spacing the phases of the four symbols in the fundamental may not be optimal because phase shifts in the higher harmonics may overlap. In principle, different phases can be chosen to maximize the "distance" between the four symbols, although this requires knowledge of the harmonic content at the receiver, which changes due to natural conditions and as the ELF waves propagate through the Earth-ionosphere waveguide.

[8] Fortunately, the optimal receiver implementation is independent of the choice of the four symbols used by the transmitter. If the noise introduced in the signal is an additive white Gaussian noise (AWGN), then the optimal receiver (the one that minimizes the probability of an error) is a matched filter receiver. The correlation receiver is an equivalent implementation and functions by computing the correlation between each received symbol and each of the four possible symbols. The symbol with the highest correlation is deemed the one most likely to have been transmitted. The probability that a received bit will be different than a transmitted bit is the BER and is only a function of the total energy in a symbol, $E_{s}$, and the noise power spectral density, $N_{0}$, which is constant over frequency for AWGN. $E_{s}$ depends on the amplitude of the generated ELF signals, which changes dramatically with natural conditions [Jin et al., 2009] as well as the duration of the symbol. Doubling the duration of the symbol period doubles $E_{s}$ at the expense of halving the transmission rate. For QPSK in AWGN, the BER has been shown to be equal to $Q\left(\sqrt{2 E_{b} / N_{0}}\right)$ [Glover and Grant, 2010], p. 424), where $E_{b}$ is the energy per bit $\left(E_{s} / 2\right.$ for QPSK) and the $Q$ function is the tail probability of the normal distribution. However, the actual ELF noise environment is substantially different from an AWGN model. Radio atmospherics (sferics) from lightning strikes are the dominant source of natural noise and have an impulsive character. Nonetheless, we will continue to use a standard correlation receiver in this article as it will simplify the analysis. However, data in both experiments and simulations throughout this article will be passed through a whitening filter before further processing. First, we take the power spectral density of a segment of VLF receiver data when HAARP was not transmitting. The filtering is performed by taking a fast Fourier transform (FFT) of the received signal (with the HAARP transmission), by dividing the spectrum by the smoothed power spectral density taken earlier, and by taking an inverse FFT. This process effectively flattens the power spectral density of both the signal and the noise in a range of $\sim 1-10 \mathrm{kHz}$, deemphasizing both the signal and the noise at frequencies with higher noise levels. This at least approximates the "white" aspect of AWGN for which the correlation receiver is optimal because components of the signal with frequencies exposed to a greater noise power have less weight after the whitening compared with components at frequencies exposed to less noise power [Lathi, 1998, p. 669]. Whitening still does not account for the impulsive aspect of the noise but is still used in past work with ELF receivers [Bernstein et al., 1974; Evans and Griffiths, 1974].

\section{Experimental Summary}

[9] The digital data transmitted using modulated HF ionospheric heating at HAARP consists of the string "Stanford
VLF" followed by a line feed (new line) encoded in 8-bit ASCII with no parity. The remainder of the data consists of random bits. Three different bit rates were used. The $100 \mathrm{bps}$ transmission lasted a total of $40 \mathrm{~s}$ and consisted of a total of 486 bytes of data. The 400 bps transmission lasts for $11 \mathrm{~s}$ and contains 536 bytes. The 800 bps transmission lasts for $5 \mathrm{~s}$ and contains 486 bytes of data. In addition to the data, each transmission is prepended with a header including a 13-bit binary phase shift keying (BPSK)modulated sequence to aid in timing synchronization as well as an equalization sequence consisting 80 consecutive 0 bits to establish a phase reference. Together, the header and the data form a packet. Each packet is transmitted at ELF frequencies of 1510,2125 , and $2735 \mathrm{~Hz}$, first with sinusoidal and then with square wave modulation. The three ELF frequencies are chosen because they are commonly used in past HAARP ELF generation experiments, and data from these experiments can be collected to examine the waveforms and noise environment at these frequencies. Past studies have also shown that ELF frequencies near $2 \mathrm{kHz}$ result in stronger ELF generation [e.g., Stubbe et al., 1982; Papadopoulos et al., 2003]

[10] The transmissions are received at a site in Chistochina, Alaska, $37 \mathrm{~km}$ northeast of HAARP using a Stanford AWESOME receiver [Cohen et al., 2010b]. Signals from two orthogonal loop antennas are sampled at $100 \mathrm{kHz}$ and digitized with 16 bits of resolution. A GPS disciplined oscillator allows sampling with $<200 \mathrm{~ns}$ timing error. For these experiments, only signals from the magnetic east-west oriented antenna are used. After the experiment, the digitized data are processed with a software decoder implemented in MATLAB.

[11] To decode the transmissions, the equalization sequence is analyzed by taking an FFT of the entire sequence and extracting the coefficients corresponding to the fundamental ELF frequency as well as four additional harmonics. The transmission can be decoded with fewer harmonics or only the fundamental, in which case only the FFT coefficients corresponding to the desired frequencies are kept. The inverse FFT of the equalization sequence is taken to obtain a time domain waveform that corresponds to the symbol 00 . The waveform is then shifted by a quarter of a period, half a period, and three quarters of a period to obtain the waveform corresponding to the other symbols. The rest of the receiver is implemented as a correlation receiver, where the correlation between the waveform corresponding to a data symbol and each of the four waveforms derived from the equalization sequence is computed. The one with the highest correlation corresponds to the symbol received. After decoding, the received bits are compared with the bits actually transmitted. The number of erroneous bits divided by the number of bits received is the BER.

[12] The signal energy $E_{b}$ is also computed from the signal and only includes the energy in the harmonics actually used to decode the transmission. For example, if only three harmonics are used to decode the transmission, $E_{b}$ includes only the energy contained in the first three harmonics. In this work, the first harmonic is synonymous with the fundamental. $N_{0}$ is always determined as the average power spectral density between 1.5 and $4.0 \mathrm{kHz}$, where the noise is primarily Gaussian and power spectral density reasonably flat. For consistency, $N_{0}$ is determined with this frequency range even when the transmission is being decoded using 
harmonics with frequencies greater than $4.0 \mathrm{kHz}$. In practice, changes in the noise level and $N_{0}$ are small compared with changes in $E_{b}$, and variations in $E_{b} / N_{0}$ are dominated by different generation conditions leading to different signal strengths.

[13] Experiments were conducted in 2011 on July 19 0932-1000 UT, July 20 0831-0900 UT, July 21 0901-0929 UT, July 24 0601-0630 UT, July 26 0729-0759 UT, and July 27 0658-0759 UT and 0802-0832 UT.

[14] The results of these experiments are shown in Figure 2 for the 3 bit rates and 3 ELF frequencies used. Each plot shows the BER as a function of $E_{b} / N_{0}$ for both sine and square wave modulation where each point represents the measured error rate in one received packet, and "No errors" indicate that no errors were present. The area surrounding each line represents a 95\% confidence interval around the estimated BER. This interval assumes that errors follow a binomial distribution in each packet and is based only on the number of errors, not on variability such as one packet randomly suffering from more sferics than another. Only the fundamental is used to decode the signal. Several features on these plots are apparent. Square wave modulation and the higher frequencies $(2125$ and $2735 \mathrm{~Hz})$ are more effective at generating ELF radiation, so there are more points with higher values of $E_{b} / N_{0}$. Lower bit rates also have higher values of $E_{b} / N_{0}$ because each symbol is transmitted for a longer period. However, this also means that each packet (which contains similar numbers of bits regardless of the bit rate) takes longer to transmit. If the ionosphere is highly variable during the $40 \mathrm{~s}$ it takes to transmit a packet at $100 \mathrm{bps}$, then the known waveform used as a phase reference may not be accurate for the duration of the transmission. This results in the occasional outliers at $100 \mathrm{bps}$ where the error rate is much higher than expected because there is more time for the phases of the received signal to drift.

[15] Figure 3 shows an example of how the BER changes when using the first three harmonics to decode a $1510 \mathrm{~Hz}$
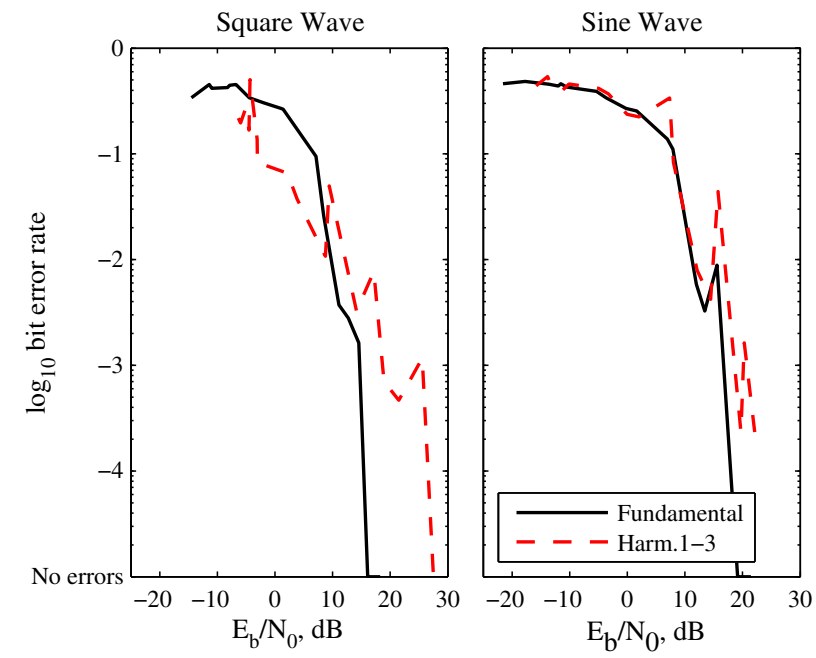

Figure 3. BER versus $\boldsymbol{E}_{\boldsymbol{b}} / \boldsymbol{N}_{\mathbf{0}}$ for a $1510 \mathrm{~Hz}$ ELF frequency at 400 bps when using only the fundamental (black solid) and when using the first three harmonics (red dashed). BER improves when using additional harmonics for square wave modulation when $\boldsymbol{E}_{\boldsymbol{b}} / \boldsymbol{N}_{\mathbf{0}}$ is low but makes little difference for sine wave modulation.

signal at 400 bps. For square wave modulation, the red dashed line representing the use of three harmonics is shifted by $5-10 \mathrm{~dB}$ to the right, indicating the larger signal energy provided by the extra harmonics. When compared with the BER from using only a fundamental with the same energy, the BER improves when $E_{b} / N_{0}$ is less than $10 \mathrm{~dB}$ but worsens at higher values of $E_{b} / N_{0}$. This indicates that having energy in the higher harmonics is better than having it in the fundamental for low values of $E_{b} / N_{0}$, possibly due to the interference in the $1510 \mathrm{~Hz}$ fundamental from a harmonic of $60 \mathrm{~Hz}$ power line noise at $1500 \mathrm{~Hz}$. For higher values of $E_{b} / N_{0}$, the additional energy is unnecessary and may be compromised by additional impulsive noise from sferics at

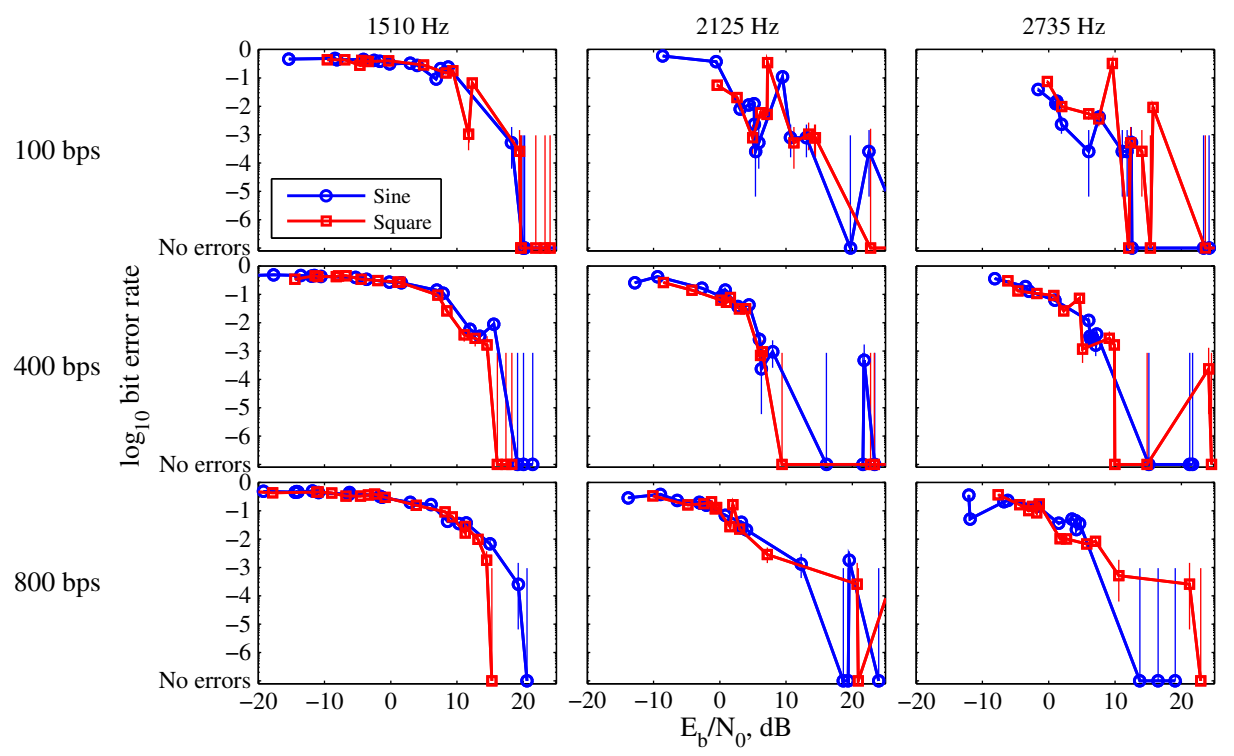

Figure 2. BER versus $\boldsymbol{E}_{\boldsymbol{b}} / \boldsymbol{N}_{\mathbf{0}}$ for ELF frequencies of 1510,2125 , and $2735 \mathrm{~Hz}$ and bit rates of 100, 400, and 800 bps when decoding using only the fundamental. Square wave modulation, higher frequencies, and lower bit rates generally result in higher values of $\boldsymbol{E}_{\boldsymbol{b}} / \boldsymbol{N}_{\mathbf{0}}$, but the curves are otherwise similar. 
higher frequencies. With sine wave modulation, the harmonics are much weaker and including them does not appreciably change the BER.

\section{Simulations}

[16] Because of limited transmission time at HAARP, the experimental results are limited for comparing transmission using one set of parameters to those at another or for comparing to theoretical models of BER performance. A $28 \mathrm{~min}$ block contains only two repetitions of a specific, modulation type, ELF frequency, and bit rate. Over the course of the entire campaign, only 16 repetitions of approximately 4000 bits means a total of 64000 bits transmitted using a particular combination of parameters. This is not sufficient to deduce BER below $10^{-4}$ as will occur for very high signal-to-noise ratios. Thus, it is helpful to develop a simple simulation of this communications system to provide better BER statistics.

[17] To simulate the received ELF signal, a typical waveform is extracted from data received at past HAARP campaigns and phase shifted appropriately to encode the data. For example, to simulate transmission using square wave modulation, we use data from an experiment on 2 April 2011, where 2 s square wave pulses were transmitted at HAARP and received at Chistochina under strong generation conditions. One period of the received waveform can then be extracted by dividing the received signal into segments one ELF period in length and averaging all the segments. This will keep any components that are periodic while averaging away some of the noise. Alternatively, an FFT of the $2 \mathrm{~s}$ pulse can be taken, keeping only the terms representing frequencies at integer multiples of the ELF frequency. An inverse FFT can then be taken to obtain a time domain waveform. This canonical waveform is then used to encode the signal in simulations. For example, to simulate transmission of the bits 01 , the derived waveform is time shifted by a quarter of a period. Once the encoding is complete, the entire signal is then scaled to represent stronger or weaker generation resulting in different signal-to-noise ratios. This simulation ignores all heating physics and propagation effects. Waveforms at different phase shifts are simply concatenated together to represent different symbols, leading to sharp discontinuities that are not physical. However, the heating and cooling time constants of the ionospheric plasma are no more than $\sim 100 \mu \mathrm{s}$, which is a small fraction of the symbol period of $2.5 \mathrm{~ms}$ even at $800 \mathrm{bps}$, so we assume that effects at the symbol boundaries are small. This simulation also does not account for changes in the ionosphere that may occur over the course of the transmission, such as generation strengthening or weakening, the harmonic content changing, or the phase of the received signal drifting due to ionospheric changes.

[18] To simulate the VLF noise environment, actual data from a VLF receiver are also used. A half hour block taken from the campaign on 22 July 2011 at 1100 UT is used because HAARP was not transmitting, but the period immediately followed experiments from the campaign. A random segment of this block is added to the scaled, encoded signal. Both the signal and the noise are sampled at $100 \mathrm{kHz}$. The resulting sum of signal and noise is then passed to the same software decoder used to decode signals from the actual experiment. The decoder outputs the BER, and the simulation can be run as many times as necessary to obtain reliable statistics.

[19] Figure 4 shows simulated BERs for transmissions at 400 bps using square wave modulation at ELF frequencies of 1510 and $2125 \mathrm{~Hz}$ with varying numbers of harmonics used. For the $1510 \mathrm{~Hz}$ case, using two harmonics improves the BER by a factor of 2-7 for $E_{b} / N_{0}$ between 7 and 15 $\mathrm{dB}$. Using three harmonics results in a dramatic improvement, where the BER improves by nearly a factor of 40 for $E_{b} / N_{0}$ of $5 \mathrm{~dB}$. Data from the actual experiment when using one and three harmonics are indicated by the dots and show a more modest improvement in BER when using three harmonics. The BER when using three harmonics is actually worse than using only the fundamental for $E_{b} / N_{0}$ greater than $15 \mathrm{~dB}$. When the received signal is very strong, the error rates are already very low. Using additional harmonics at higher frequencies may expose the received signal to greater levels of impulsive noise, which worsens the BER in this situation.

[20] At $2125 \mathrm{~Hz}$, using additional harmonics results in only a slight improvement in BER for $E_{b} / N_{0}<3 \mathrm{~dB}$, while still worsening the BER for the stronger signals. Additional
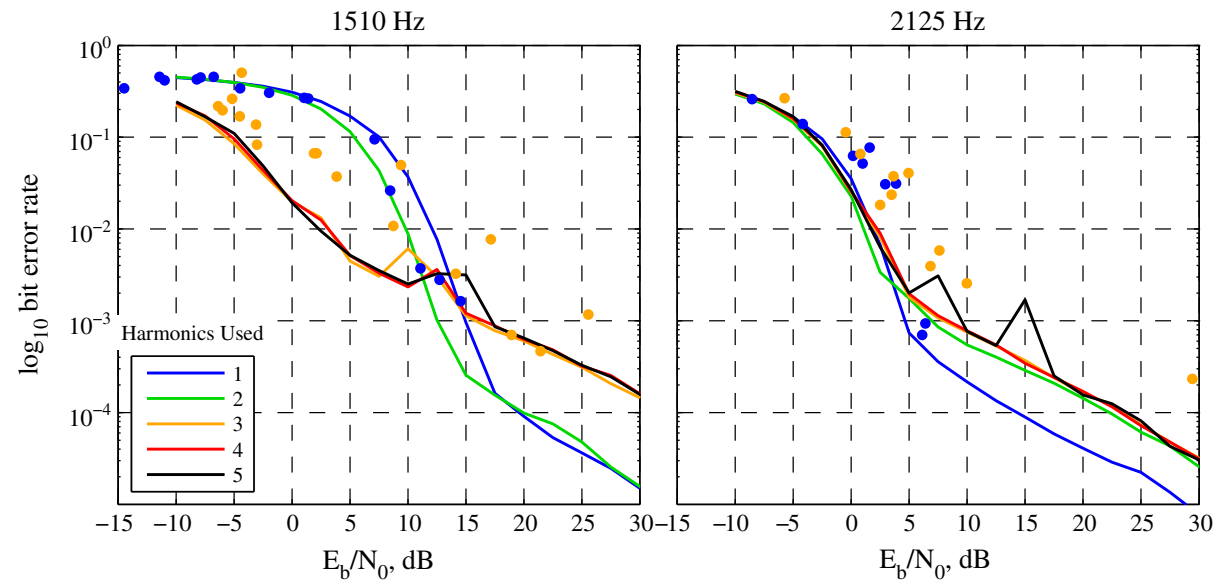

Figure 4. BER versus $\boldsymbol{E}_{\boldsymbol{b}} / \boldsymbol{N}_{\mathbf{0}}$ for 1510 and $2125 \mathrm{~Hz}$ ELF frequencies at $400 \mathrm{bps}$ when using different numbers of harmonics. At $1510 \mathrm{~Hz}, \mathrm{BER}$ improves for smaller values of $\boldsymbol{E}_{\boldsymbol{b}} / \boldsymbol{N}_{\mathbf{0}}$ when using up to three harmonics. At $2125 \mathrm{~Hz}$, BER only improves after adding the second harmonic. For comparison, dots represent experimental data when using one and three harmonics. 


\section{JIN ET AL.: HAARP NONLINEARITY IN COMMUNICATIONS}

harmonics are more beneficial to the $1510 \mathrm{~Hz}$ transmission for several reasons. ELF generation at $1510 \mathrm{~Hz}$ is weaker than at $2125 \mathrm{~Hz}$, and the $1510 \mathrm{~Hz}$ signal receives greater interference from the very strong 25 th harmonic of $60 \mathrm{~Hz}$ power line noise at $1500 \mathrm{~Hz}$. Square wave modulation results in a very strong third harmonic component, which suffers much less power line interference and reduces the BER dramatically, whereas the weaker second harmonic only contributes to a small improvement in BER. As the number of harmonics increases beyond three, the additional higher harmonics become weaker, in addition to being in a stronger noise environment. Figure 1 shows the impulsive noise increases beyond $\sim 4.5 \mathrm{kHz}$. Thus, the fourth and higher harmonics of the $1510 \mathrm{~Hz}$ transmission and the third and higher harmonics of the $2125 \mathrm{~Hz}$ transmission have poor signal-to-noise ratios and do not contribute to further reductions in the BER.

\section{Theoretical Performance Models}

[21] To characterize the BER of this ELF communications system, we would like to fit mathematical expressions for BER developed in past work to this new system with the complication that the characteristics of the noise changes as harmonics at higher frequencies are added. A closed form expression for the BER makes it much faster to iterate and optimize over different parameters than repeatedly running large simulations. To examine a simplified version of this problem, we will consider only the fundamental and third harmonic of a $2125 \mathrm{~Hz}$ signal. The fundamental is received very strongly and is at a frequency that is not strongly affected by either $60 \mathrm{~Hz}$ power line harmonics and only affected by strong nearby sferics. The third harmonic at $6375 \mathrm{~Hz}$, however, is subject to primarily impulsive noise from sferics.

[22] A QPSK system is easier to analyze as two independent BPSK systems. BPSK uses only two phases to transmit one bit (e.g., $x_{0}(t)=-\cos (2 \pi f t)$ and $\left.x_{1}(t)=\cos (2 \pi f t)\right)$, where $x_{0}(t)$ and $x_{1}(t)$ are the symbols representing a 0 and 1 bit. Because the sine and the cosine functions are orthogonal, a second BPSK signal using $\sin (2 \pi f t)$ and $-\sin (2 \pi f t)$ can be transmitted on the same channel without interference, and the BER of QPSK is actually the same as for BPSK. The same holds true if the symbol is a combination of odd harmonics, such as $x_{0}(t)=\cos (2 \pi f t)+b \cos (6 \pi f t+\varphi)$, where $b$ and $\varphi$ are the amplitude and phase of the third harmonic relative to the first. For a received signal $y=x(t)+n(t)$, where $n(t)$ is the noise, the output of the correlator is:

$$
\begin{aligned}
& \frac{1}{T} \int_{0}^{T} x_{1}(t) y(t) \mathrm{d} t \\
= & E+\frac{1}{T} \int_{0}^{T} x_{1}(t) n(t) \mathrm{d} t
\end{aligned}
$$

where $E$ is the energy in the signal. Thus, an error occurs when the noise term is less than $-E$ because this would cause the correlator output to be the wrong sign and the decision would be for the incorrect bit. The error probability is determined from the probability density function (PDF) of the noise term, which can be integrated to determine the probability of the noise term being greater than $E$. To estimate the PDF, we examine the output when noise is injected into the correlator. Figure 5 shows the PDF of the output of three correlators when 20,000 segments of ELF/VLF noise 5 $\mathrm{ms}$ in length (corresponding to a $400 \mathrm{bps}$ bit rate) are used as input. The correlators are matched to a typical $2125 \mathrm{~Hz}$ ELF signal generated by HAARP using only the fundamental, only the third harmonic, and both the fundamental and third harmonic. At the fundamental, the PDF closely resembles a Gaussian (gray dotted line) until values greater than approximately $10^{5}$ where a heavy tail begins. This tail represents the presence of rare but strong sferics that add an impulsive component to the otherwise largely Gaussian noise. A mixture of Gaussian and impulsive noise was modeled by Ziemer [1967], and the resulting PDF was reproduced as follows:

$$
\begin{aligned}
p_{1}(n)= & (1-\gamma) \frac{e^{-n^{2} /\left(2 \sigma_{G}^{2}\right)}}{\sqrt{2 \pi \sigma_{G}^{2}}} \\
& +2\left(\frac{\gamma}{\pi}\right)^{3 / 2} \int_{0}^{\pi / 2} \frac{\exp \left[\frac{-\gamma n^{2}}{2\left(\gamma \sigma_{G}^{2}+2 \sigma_{T}^{2} \cos ^{2}\right)}\right]}{\sqrt{2\left(\gamma \sigma_{G}^{2}+2 \sigma_{I}^{2} \cos ^{2} y\right)}} d y
\end{aligned}
$$

where $\sigma_{G}^{2}$ and $\sigma_{I}^{2}$ are the variances of the Gaussian and impulsive components of the noise, $n$, and $\gamma$ is the average number of noise impulses occurring during one symbol period.
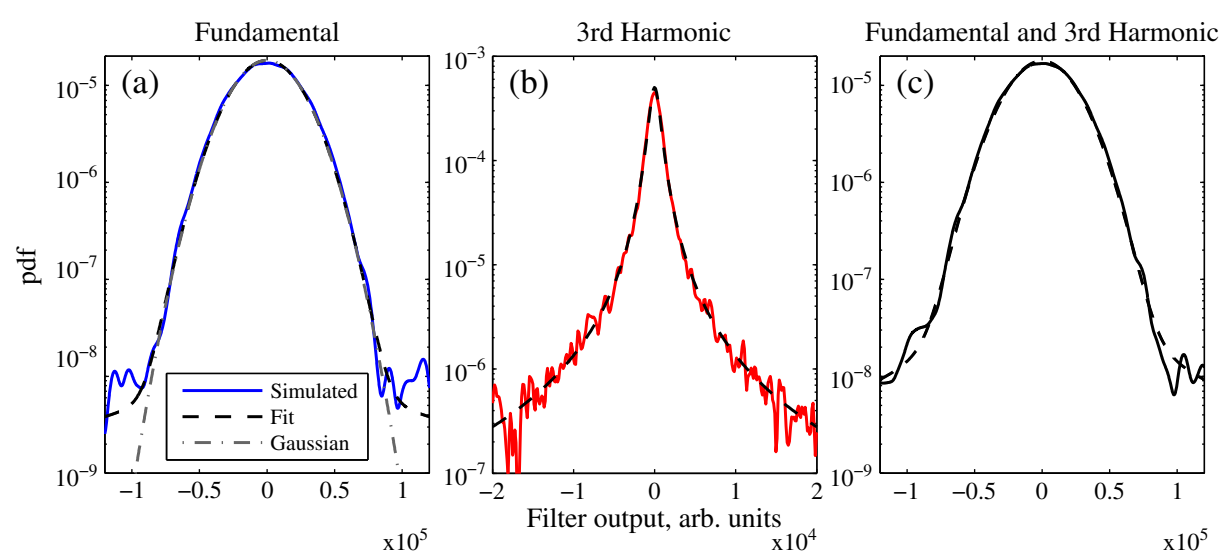

Figure 5. PDF at output of a $2125 \mathrm{~Hz}$ matched filter derived from 20,000 segments of VLF noise $5 \mathrm{~ms}$ long and a fit using mathematical models in the dashed lines. 
Values of $\sigma_{G}=22000, \sigma_{I}=6000$, and $\gamma=2.6 \times 10^{-3}$ fit the simulated PDF well and is shown as the dotted black line.

[23] The PDF of the correlator using only the third harmonic at $6375 \mathrm{~Hz}$ is clearly non-Gaussian and very heavy tailed. The third harmonic is also weaker than the fundamental, so the scale on the $x$-axis is smaller. We found that the generalized " $t$ " model described by Hall [1966, p. 16] provided a better fit in this case:

$$
p_{3}(n)=\frac{\Gamma(\theta / 2)}{\Gamma((\theta-1) / 2)} \frac{\beta^{\theta-1}}{\sqrt{\pi}\left(n^{2}+\beta^{2}\right)^{\theta / 2}}
$$

where $\Gamma$ is the gamma function, $\beta$ is a parameter specifying the width of the distribution, which is related to the total noise energy, and $\theta$ is a parameter specifying the slope of the tail of the distribution. Values of $\beta=750$ and $\theta=2.282$ were used to fit this distribution to simulations in Figure $5 \mathrm{~b}$ and are consistent with the results of Hall [1966] where values of $\theta$ between 2 and 4 fit measured data.

[24] A correlator that incorporates both the fundamental and the third harmonic is equivalent to summing the output of the correlators using only the fundamental and only the third harmonic. The PDF of the output of this correlator is given by the convolution of the PDFs of the correlator using the individual harmonics (equations 3 and 4), assuming the two noise processes are independent. The noise processes occurring at the fundamental and third harmonic frequencies are not independent as a strong sferic that appears at the fundamental will likely also appear in the third harmonic. However, given that strong harmonics in the fundamental are infrequent $(\gamma \ll 1)$, then it is reasonable to expect that the primarily Gaussian noise at the fundamental frequency is independent of the large amount of impulsive noise at the third harmonic frequency. Figure $5 \mathrm{c}$ shows the PDF of the correlator using both the fundamental and the third harmonic, as well as the PDF obtained by convolving equations (3) and (4). The behavior of this PDF is very similar to that using only the fundamental because the fundamental is stronger and thus weighted more. However, the tails of the distribution are elevated because of the large impulsive component from the third harmonic.

[25] The BER can be computed by integrating the PDF to find the probability that the noise at the matched filter output will be greater than the energy in the signal:

$$
P_{e}=\int_{E}^{\infty} p(n) \mathrm{d} n
$$

where $P_{e}$ is the probability of error, or BER, and $p(n)$ is the noise PDF at the correlator output. The resulting BERs computed by integrating equations (3) and (4) and their convolution are shown in Figure 6 together with the BERs from simulations conducted as in section 4. Although there are discrepancies between the two methods of determining the BER that could be the result of inconsistent calculations of the signal energy, the BER computed from the PDFs are still able to reproduce the overall behavior seen in the simulations. Using both the first and the third harmonic results in a worse BER compared with using only a fundamental with the same energy as the first and third harmonic. This is especially noticeable at higher values of $E_{b} / N_{0}$ where impulsive noise becomes the
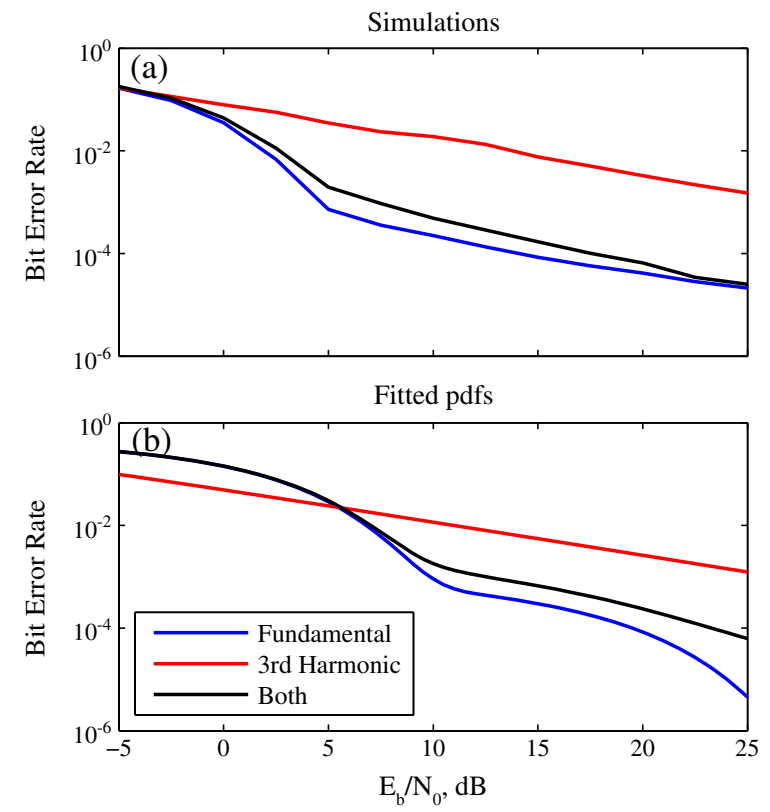

Figure 6. BERs computed from $2125 \mathrm{~Hz}$ simulation (a) and from integrating expressions of the noise PDF (b). Adding the third harmonic generally results in a worse BER compared with using only the fundamental with the same signal energy. The BER from the fitted PDFs is able to reproduce the behavior in the simulations.

dominant source of errors and the noise in the third harmonic is largely impulsive.

\section{Conclusion}

[26] Experiments were conducted at HAARP, where QPSK-modulated signals were used to transmit data using three ELF frequencies $(1510,2125$, and $2735 \mathrm{~Hz})$ and using both sine and square wave modulation. We find that BERs are lower for square wave modulation at 2125 and 2735 $\mathrm{Hz}$, which generates stronger transmitted signals. Using the strong harmonics generated using square wave modulation further reduced the BER when the signal was weak. When including higher harmonics, the greatest reduction in BER occurred at a frequency of $1510 \mathrm{~Hz}$ as the fundamental is weaker and exposed to more interference from power lines. However, simulations of this communication system using waveforms and noise derived from data showed that using additional harmonics only lowers the BER when the harmonics are below $\sim 4.5 \mathrm{kHz}$. Impulsive noise from sferics is the dominant source of errors at higher frequencies and for stronger signals and limits the BER performance in these regimes. The performance of a receiver using a fundamental below $4.5 \mathrm{kHz}$ and a third harmonic above can be modeled by combining existing mathematical expressions for the noise distribution at these frequencies. This model can reproduce the BER features observed in simulations that show the inclusion of higher harmonics will not improve the BER when the higher harmonics are exposed to more impulsive noise.

[27] Acknowledgments. This research has been carried out with support from HAARP, Office of Naval Research, Air Force Research 


\section{JIN ET AL.: HAARP NONLINEARITY IN COMMUNICATIONS}

Laboratory, and Defense Advanced Projects Research Agency, via ONR grant nos. N0001405C0308 and N00014091 to Stanford University and by a Stanford Graduate Fellowship. The authors thank Mike McCarrick and Helio Zmwi for operating the HAARP array as well as Ed Kennedy for organizing the experimental campaign that led to these results.

\section{References}

Barr, R., and P. Stubbe (1984), ELF and VLF radiation from the "polar electrojet antenna", Radio Sci., 19, 1111-1122, doi:10.1029/RS019i004p01111.

Bernstein, S. L., M. L. Burrows, J. E. Evans, A. S. Griffiths, D. A. McNeill, C. W. Niessen, I. Richer, D. P. White, and D. K. Willim (1974), Longrange communications at extremely low frequencies, Proc. IEEE, 62(3), 292-312, doi:10.1109/PROC.1974.9426.

Budden, K. G. (1961), The Wave-Guide Mode Theory of Wave Propagation, Prentice-Hall, Englewood Cliffs, NJ.

Cohen, M. B., U. S. Inan, M. Gołkowski, and M. J. McCarrick (2010a), ELF/VLF wave generation via ionospheric HF heating: Experimental comparison of amplitude modulation, beam painting, and geometric modulation, J. Geophys. Res. Space Physics, 115(A14), 2302-+, doi:10.1029/2009JA014410.

Cohen, M. B., U. S. Inan, and E. W. Paschal (2010b), Sensitive Broadband ELF/VLF Radio Reception With the AWESOME Instrument, IEEE Trans. Geosci. Remote Sens., 48, 3-17, doi:10.1109/TGRS.2009.2028334.

Evans, J. E., and A. S. Griffiths (1974), Design of a sanguine noise processor based upon world-wide extremely low frequency (elf) recordings, IEEE Trans. Commun., 22(4), 528-539, doi:10.1109/TCOM.1974.1092215.

Getmantsev, G. G., N. A. Zuikov, D. S. Kotik, L. F. Mironenko, N. A. Mitiakov, V. O. Rapoport, I. A. Sazonov, V. I. Trakhtengerts, and V. I. Eidman (1974), Combination frequencies in the interaction between high-power short-wave radiation and ionospheric plasma, Sov. JETP Lett., 20, 229-232.

Glover, I. A., and P. M. Grant (2010), Digital Communications, 3rd ed., Pearson. Gołkowski, M., U. S. Inan, A. R. Gibby, and M. B. Cohen (2008), Magnetospheric amplification and emission triggering by ELF/VLF waves injected by the 3.6 MW HAARP ionospheric heater, J. Geophys. Res. Space Physics, 113, A10201, doi:10.1029/2008JA013157.

Hall, H. M. (1966), New model for impulsive phenomena: application to atmospheric noise communication channels, Tech. rep., Stanford Electronics Laboratories.

Ingram, R. (1984), Performance of the locally optimum threshold receiver and several suboptimal nonlinear receivers for elf noise, IEEE J. Oceanic Eng., 9(3), 202-208, doi:10.1109/JOE.1984.1145615.
James, H. G. (1985), The ELF spectrum of artificially modulated D/E-region conductivity, J. Atmos. Terr. Phys., 47, 1129-1142.

Jin, G., M. Spasojevic, and U. S. Inan (2009), Relationship between electrojet current strength and ELF signal intensity in modulated heating experiments, J. Geophys. Res. Space Physics, 114(A13), 8301-+, doi:10.1029/ 2009JA014122.

Jin, G., M. Spasojevic, M. B. Cohen, and U. S. Inan (2012), Harmonic minimization waveforms for modulated heating experiments at HAARP, J. Geophys. Res. Space Physics, 117, A11315.

Kapustin, I. N., R. A. Pertsovskiǐ, A. N. Vasil'Ev, V. S. Smirnov, O. M. Raspopov, L. E. Solov'eva, A. A. Ul'Yachenko, A. A. Arykov, and N. V. Galakhova (1977), Generation of radiation at combination frequencies in the region of the auroral electric jet, Sov. JETP Lett., 25, 228-231.

Kassam, S. A. (1988), Signal Detection in Non-Gaussian Noise, SpringerVerlag, New York.

Lathi, B. P. (1998), Modern digital and analog communication systems, Oxford University Press, New York.

Milikh, G. M., K. Papadopoulos, M. McCarrick, and J. Preston (1999), ELF emission generated by the HAARP HF-heater using varying frequency and polarization, Radiophys. Quantum Electron., 42, 639-646, doi:10.1007/BF02676849.

Moore, R. C., U. S. Inan, and T. F. Bell (2006), Observations of amplitude saturation in ELF/VLF wave generation by modulated HF heating of the auroral electrojet, Geophys. Res. Lett., 33, L12106, doi:10.1029/ 2006GL025934.

Papadopoulos, K., T. Wallace, M. McCarrick, G. M. Milikh, and X. Yang (2003), On the efficiency of ELF/VLF generation using HF heating of the auroral electrojet, Plasma Phys. Rep., 29, 561-565.

Rietveld, M. T., H.-P. Mauelshagen, P. Stubbe, H. Kopka, and E. Nielsen (1987), The characteristics of ionospheric heating-produced ELF/VLF waves over 32 hours, J. Geophys. Res., 92, 8707-8722.

Stubbe, P., H. Kopka, M. T. Rietveld, and R. L. Dowden (1982), ELF and VLF wave generation by modulated HF heating of the current carrying lower ionosphere, J. Atmos. Terr. Phys., 44, 1123-1131.

Tomko, A. A. (1981), Nonlinear phenomena arising from radio wave heating of the lower ionosphere, Ph.D. thesis, Pennsylvania State Univ., University Park, PA.

Velikhov, E., A. Zhamaletdinov, A. Shevtsov, A. Tokarev, Y. Kononov, L. Pesin, G. Kadyshevich, M. Pertel, and A. Veshchev (1998), Deep electromagnetic studies with the use of powerful ELF radio installations, Izv. Phys. Solid Earth, 34, 615-32.

Ziemer, R. (1967), Error probabilities due to additive combinations of gaussian and impulsive noise, IEEE Trans. Commun. Technol., 15(3), 471-474, doi:10.1109/TCOM.1967.1089608. 